\title{
AVALIAÇÃO DE MÉTODOS DE CÁLCULO QUANTO ÀS CONTRIBUIÇÕES DO REFORÇO AO CORTANTE DE VIGAS DE CONCRETO ARMADO ATRAVÉS DE COLAGEM EXTERNA POR COMPÓSITOS DE CFRP
}

\author{
Calculation Methods Assessment on the Contributions of Shear \\ Strengthening of Reinforced Concrete Beams by Externally Bonding \\ CFRP Composites
}

Nicolás Roa Rojas ${ }^{1}$, Nívea Gabriela Benevides de Alburqueque ${ }^{2}$, Guilherme Sales Soares de Azevedo Melo $^{3}$, Nathaly Sarasty Narváez ${ }^{4}$

Recebido em 17 de agosto de 2016; recebido para revisão em 21 de novembro de 2016; aceito em 24 de março de 2017; disponível on-line em 30 de abril de 2017.

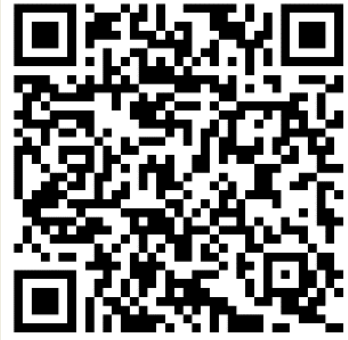

PALAVRAS CHAVE:

Reforço ao cisalhamento; Interação fibra-concreto; Modelos teóricos; Colagem exerna.

\section{KEYWORDS:}

Shear reinforcement; Fiber-stirrups interaction; Theoretical models; External bonding.

RESUMO: Este trabalho trata da análise de vigas de concreto armado reforçadas ao cortante através da colagem externa de compósitos de fibras poliméricas (EB-FRP), tomando-se por base os modelos teóricos propostos por autores como Chen et al. (2003) e Chen (2010) - cujas abordagens fundamentam-se na análise de resultados experimentais e computacionais - sendo contrastadas às previsões das contribuições estimadas por normas vigentes. Dessa forma, buscou-se realizar uma avaliação dos dados e modelos de cálculo sugeridos pelos autores, salientando a influência de parâmetros particulares nas capacidades dos reforços que geram resultados mais próximos aos obtidos experimentalmente. Nesta pesquisa também foi analisada a precisão das estimativas das contribuições dos reforços, em especial das fibras de carbono, determinadas pelos modelos e pela norma americana $\mathrm{ACl} 440.2 \mathrm{R}$ (2008) e italiana CNR DT200 (2004) em relação aos resultados de um banco de dados experimental selecionado, os quais conduzem a resultados ainda pouco precisos. Isto é atribuído principalmente a não consideração das interações existentes entre estribos e fibras de carbono como materiais de reforço, como também de algumas propriedades físicas, mecânicas e geométricas. Diante disso, constata-se a necessidade contínua de refinamento dos modelos teóricos para a obtenção de resultados mais satisfatórios.

ABSTRACT: This work deals with the analysis of reinforced concrete beams strengthened to shear force by using externally bonded fiber reinforced polymer composites (EB-FRP), based upon the theoretical models proposed by authors such as Chen et al. (2003) and Chen (2010) - whose approaches are grounded on the analysis of experimental and computational results - and contrasted to the provisions of estimated contributions by current codes. Thus, it sought to conduct an assessment of the data and calculation models suggested by the authors, emphasizing the influence of particular parameters on reinforcement capabilities that generate results closer to those obtained experimentally. The accuracy of estimations, especially the carbon fiber's contribution, is also analyzed through the American code ACI 440.2R (2008) and the Italian code CNR DT200 (2004) regarding selected experimental databases, which lead to results still not accurate. This is mainly attributed to non consideration of interactions between stirrups and fibers as reinforcing materials, as well as some physical, mechanical and geometrical properties. Thus, there has been a continued need for refinement of numerical and theoretical models to obtain more satisfactory results.

\footnotetext{
* Contato com os autores:

${ }^{1}$ e-mail: roarojasnicolas@gmail.com ( N. R. Rojas)

Engenheiro Civil, Mestrando do Prog. de Pós-Graduação em Estruturas e construção Civil (PECC) da Universidade de Brasília (UnB)

2e-mail: niveagabriela@hotmail.com ( N. G. B. Albuquerque)

Engenheira Civil, Pós-doutoranda, Universidade de Brasília (UnB)

${ }^{3}$ e-mail: melog@unb.br ( G. S. Melo)

Engenheiro Civil, Professor Doutor, Universidade de Brasília (UnB)

${ }^{4}$ e-mail: natalysarasty@hotmail.com ( N. S. Narváez )

Engenheira Civil, Doutoranda do Prog. de Pós-Graduação em Estruturas e construção Civil (PECC) da Universidade de Brasília (UnB)
} 


\section{INTRODUÇÃO}

Conforme o fib Bulletin 14 (2001), a necessidade do reforço estrutural pode dever-se a muitas circunstâncias, tais como: o tempo de serviço da estrutura, falta de manutenção, dano acidental ou natural, solicitações adicionais não previstas na etapa de projeto, degradação por más condições das armaduras ou baixa qualidade dos materiais utilizados, entre outras. Frente a essa deterioração das estruturas, houve um desenvolvimento vertiginoso de suas técnicas de reparo, reabilitação e reforço, destacando-se neste trabalho as fibras de reforço polimérico (FRP), dada sua alta efetividade e vasta aplicação. Atualmente, embora pesquisas venham sendo desenvolvidas na área, o Brasil ainda carece de norma regulamentadora que auxilie os projetistas no dimensionamento e análise do sistema estrutural de $\mathrm{FRP}$, os quais, em geral, recorrem a modelos analíticos de normas internacionais, tais como as referidas no fib Bulletin 14 (2001), de onde se destacam as versões mais recentes das normas $\mathrm{ACl}$ 440.2R (2008) e CNR-DT200 (2004).

A metodologia de colagem externa, "external bonding", de fibras de reforço polimérico (EB-FRP) é geralmente aplicada visando o incremento de capacidade à flexão e ao cisalhamento de elementos estruturais. Contudo, o principal problema do sistema encontra-se relacionado à superfície de aderência entre concreto e fibra de carbono), que leva à descolagem da fibra de carbono (Mofidi e Chaallal, 2011; Adhikary et al., 2004; Kim et al., 2013; Kim et al., 2014), sendo este o principal tipo de falha estrutural do sistema de colagem externa. Deve-se atentar, portanto, que sempre que em um elemento estrutural a resistência ao cisalhamento estiver abaixo à de flexão haverá a necessidade urgente de se realizar adequadamente o reforço, a fim de evitar um possível colapso súbito que caracteriza a ruptura por cisalhamento pelas condições de insegurança e risco apresentadas (Bousselham e Chaallal, 2004). Nesse cenário, a solução pode se apresentar através de reforços contínuos (através de mantas de fibra de carbono) ou não contínuos (através de faixas de fibra de carbono), sendo este último o mais eficiente, uma vez que não transferem deformações nem problemas de descolagem de um faixa para a outra, o que lhes permite trabalhar de maneira independente. As faixas podem ser dispostas em diferentes formatos, como: fechado ou "wrapped" (envolve totalmente a peça), semiaberto (em forma de "U") e o lateral (reforço das faces laterais da viga).

O reforço EB-FRP é executado através da colagem e sobreposição de fibras de carbono coladas à superfície de concreto que, por sua vez, mudam a rigidez axial da peça, um dos parâmetros que devem ser levados em conta na implementação de modelos teóricos. Além deste efeito, pesquisas recentes (Mofidi e Chaallal, 2011; Chen, 2010; Bousselham e Chaallal, 2004; Chen et al., 2013) avaliam outros parâmetros no desempenho da fibra de carbono como reforço ao cisalhamento devido à interação que existe entre concreto, aço e fibra de carbono, e alguns parâmetros geométricos como a relação geométrica do vão $(\mathrm{a} / \mathrm{l})$, taxa do reforço longitudinal, efeito de tamanho, o comprimento efetivo de ancoragem, entre outros, os quais ainda não são considerados nos modelos de cálculo das normas atuais, gerando estimativas conservadoras.

Este trabalho faz uma revisão dos modelos de estimativa das contribuições de resistência ao cortante por sistemas de colagem externa de FRP mais particularmente fibras de carbono, CFRP abordados por prescrições normativas vigentes e outros propostos por autores, avaliando-se a precisão com respeito a resultados experimentais obtidos por meio de uma base de dados coletados da literatura cientifica disponível atualmente.

\section{CONTRIBUIÇÕES À RESISTÊNCIA AO CORTANTE ATRAVÉS DE SISTEMAS DE REFORÇO EB-FRP}

O reforço ao cortante de elementos estruturais com sistemas de EB-FRP é altamente utilizado em função de suas boas caraterísticas de resistência, durabilidade e execução (Obaidat et al., 2009; Bousselham e Chaallal, 2006; Obaidat, 2011). O comportamento à força cortante de vigas 
reforçadas de concreto armado se encontra sujeito a um grande número de fatores que influenciam na eficiência das FRP, cujos parâmetros ainda não são totalmente considerados nos modelos adotados pelas normas vigentes, e culminam consequentemente em estimativas conservadoras. Diversas pesquisas experimentais reportam que o principal tipo de falha do sistema de reforço ocorre pela descolagem da fibra (Mofidi e Chaallal, 2011; Adhikary et al., 2004; Kim et al., 2013; Kim et al., 2014), portanto, ao longo deste trabalho são consideradas as equações dos modelos sob tal critério de ruptura, onde a capacidade nominal das peças reforçadas com fibra de carbono e os diferentes modelos teóricos de estimativa são apresentados a seguir.

\subsection{RESISTÊNCIA NOMINAL DE VIGAS REFORÇADAS AO CORTANTE COM SISTEMAS EB-FRP}

De maneira geral, as normas e códigos de projeto atuais calculam a capacidade nominal ao cisalhamento de elementos estruturais reforçados com FRP como a soma das contribuições de resistência do concreto, aço e fibra de carbono, conforme Equação 1:

$$
V_{n}=V_{c}+V_{s}+V_{f}
$$

\section{Em que:}

$\boldsymbol{V}_{\boldsymbol{n}}=$ resistência nominal ao cisalhamento $(\mathrm{N})$;

$V_{c}=$ contribuição de resistência ao cisalhamento pelo concreto $(\mathrm{N})$;

$\boldsymbol{V}_{\boldsymbol{s}}=$ contribuição de resistência ao cisalhamento pelos estribos $(\mathrm{N})$;

$\boldsymbol{V}_{\boldsymbol{f}}=$ contribuição de resistência ao cisalhamento pelas FRP (N).

A premissa de superposição das contribuições de resistência ao cisalhamento por parte de cada material é questionada por diversos autores, tais como Chen e Teng (2003), Dirar (2012) e Lu et al. (2005), nos modelos teóricos de normas vigentes, em função de não considerarem fatores de redução diante dos efeitos negativos na resistência decorrentes da interação entre estribos e fibras de carbono. Bousselham e Chaallal (2006) e Pellegrino e Modena (2008) explicam que, dentre os materiais de reforço, o aço e a fibra não conseguem atingir sua contribuição máxima no mesmo instante em que o incremento das taxas de aço transversal, o que gera efeito negativo na eficiência da fibra com reforço. É, portanto, necessário considerar modelos teóricos que levem em conta este tipo de efeito com o objetivo de otimizar o projeto ao cortante de elementos estruturais reforçados com sistemas de FRP, visando estimativas menos conservadoras.

\subsection{MODELOS DE ESTIMATIVA DA CONTRIBUIÇÃO DA FIBRA DE CARBONO, Vf}

Apresentam-se, a seguir, prescrições normativas vigentes através de modelos de cálculo adotadas atualmente em contraposição aos modelos teóricos propostos em estudos recentes, cujas estimativas das contribuições de resistência ao cisalhamento pelo reforço com sistemas de CFRP levam em consideração a interação existente entre aço e fibra de carbono como reforço e, portanto, são refletidos nos parâmetros relacionados ao comportamento do reforço. As equações apresentadas para cada modelo são aplicáveis às vigas reforçadas com FRP em faixas ("strips") e em formato de " $U$ ", omitindo-se as equações para reforço fechado e lateral bem como para sistemas de reforço contínuos em mantas ("sheets"), cujas equações podem ser consultadas diretamente nos trabalhos dos autores supracitados.

\subsubsection{Norma ACl 440.2R (2008)}

O Instituto Americano do Concreto estabelece o modelo de cálculo de contribuições de resistência ao cisalhamento em elementos reforçados com FRP baseado nas pesquisas desenvolvidas na Universidade de Missouri por Khalifa et al. (1998). Considerando ruptura por descolamento, a contribuição de resistência da fibra de carbono é dada por meio das Equações 2, 3 e 4:

$$
V_{f}=\frac{A_{f v} \cdot f_{f e}(\sin \alpha+\cos \alpha) \cdot d_{f v}}{s_{f}}
$$

[2]

$$
A_{f v}=2 \cdot n \cdot t_{f} \cdot w_{f}
$$




$$
f_{f e}=\varepsilon_{f e} \cdot E_{f}
$$

Em que:

$V_{f}=$ valor da força cortante aportada pela fibra de carbono (N);

$\boldsymbol{A}_{f v}=$ área da fibra de carbono $\left(\mathrm{mm}^{2}\right)$;

$f_{f e}=$ tensão efetiva nas fibras de carbono (MPa);

$\alpha=$ ângulo de inclinação do reforço de FRP (graus);

$\boldsymbol{d}_{f v}=$ altura útil da fibra de carbono $(\mathrm{mm})$;

$\boldsymbol{s}_{f}, \boldsymbol{t}_{f}, \boldsymbol{w}_{f}=$ espaçamento, espessura e largura das FRP $(\mathrm{mm})$;

$\boldsymbol{n}=$ número de camadas da fibra de carbono;

$\boldsymbol{\varepsilon}_{f e}=$ deformação efetiva da fibra de carbono (\%o);

$\boldsymbol{E}_{f}=$ módulo de elasticidade da fibra de carbono (MPa).

A deformação efetiva nas fibras de carbono é influenciada pelo comportamento de cada tipo de reforço; para reforço em " $U$ " depende do comprimento efetivo de aderência da fibra de carbono. Uma vez que o fator $K_{v}$ é função da resistência a compressão do concreto, do sistema de reforço utilizado e da rigidez da fibra de carbono, tem-se as Equações de 5 a 9:

$$
\begin{aligned}
& \varepsilon_{f e}=K_{v} \cdot \varepsilon_{f u} \leq 0.004 \\
& K_{v}=\frac{k_{1} \cdot k_{2} \cdot L_{e}}{11,900 \cdot \varepsilon_{f u}} \leq 0,75 \\
& L_{e}=\frac{23,300}{\left(n \cdot t_{f} \cdot E_{f}\right)^{0.58}} \\
& K_{1}=\left(\frac{f_{c}^{\prime}}{4000}\right)^{2 / 3} \\
& K_{2} \cdot\left\{\frac{d_{f v}-L_{e}}{d_{f v}}\right\} \text { Para reforço aberto em "U” Eq. [9] }
\end{aligned}
$$

Em que:

$\varepsilon_{f e}=$ deformação efetiva da fibra de carbono (\%o);

$\boldsymbol{K}_{v}=$ fator de aderência que depende do cisalhamento; $\varepsilon_{f u}=$ deformação última da fibra de carbono(\%);

$\boldsymbol{d}_{f v}=$ altura útil da fibra de carbono(mm);

$\boldsymbol{L}_{e}=$ comprimento efetivo de aderência $(\mathrm{mm})$;

$\boldsymbol{k}_{1}, \boldsymbol{k}_{\mathbf{2}}=$ fatores de modificação;

$f_{c}=$ resistência à compressão do concreto (MPa).

\subsubsection{Norma CNR-DT200 (2004)}

A norma de projeto e construção de sistemas de reforço em estruturas existentes através da colagem externa de FRP é parte do código italiano e baseia-se nas pesquisas desenvolvidas pela equipe de pesquisadores de Roma e Napoli, Giorgio Monti e Antonio Nanni. A contribuição de resistência ao cortante devido ao reforço em tiras em "U" é calculada pela Equação 10:

\section{Em que:}

$\varepsilon_{f e}=$ deformação efetiva da fibra de carbono (\%o);

$\boldsymbol{K}_{\boldsymbol{v}}=$ fator de aderência que depende do cisalhamento;

$\varepsilon_{f u}=$ deformação última da fibra de carbono(\%);

$\boldsymbol{d}_{f v}=$ altura útil da fibra de carbono $(\mathrm{mm})$;

$\boldsymbol{L}_{e}=$ comprimento efetivo de aderência $(\mathrm{mm})$;

$\boldsymbol{k}_{1}, \boldsymbol{k}_{\mathbf{2}}=$ fatores de modificação;

$f_{c}=$ resistência à compressão do concreto (MPa).

A tensão efetiva da fibra de carbono estimada considerando-se falha por descolamentoé calculada através da Equação 11:

Em que:

$\boldsymbol{f}_{\text {fed }}=$ Tensão efetiva por descolamento da fibra de carbono ;

$f_{f d d}=$ resistência de descolamento de projeto para o reforço com FRP (MPa);

$l_{e}=$ comprimento efetivo de ancoragem $(\mathrm{mm})$;

$\boldsymbol{\beta}=$ ângulo de inclinação das tiras de reforço (graus);

$\boldsymbol{d}=$ altura útil $(\mathrm{mm})$;

$\boldsymbol{h}_{\boldsymbol{m}}=$ altura da viga $(\mathrm{mm})$.

Os parâmetros apresentados nas Equações 9 a 11 são apresentados graficamente na Figura 1.

$$
V_{R d, f}=\frac{1}{\gamma_{R d}} \cdot 0.9 \cdot d \cdot f_{f e d} \cdot 2 \cdot t_{f} \cdot(\cot (\theta)+\cot (\beta)) \cdot \frac{w_{f}}{p_{f}}
$$




$$
f_{f e d}=f_{f d d} \cdot\left[1-\frac{1}{3} \cdot \frac{l_{e} \cdot \operatorname{sen} \beta}{\operatorname{Min}\left\{0.9 \cdot d, h_{m}\right\}}\right]
$$
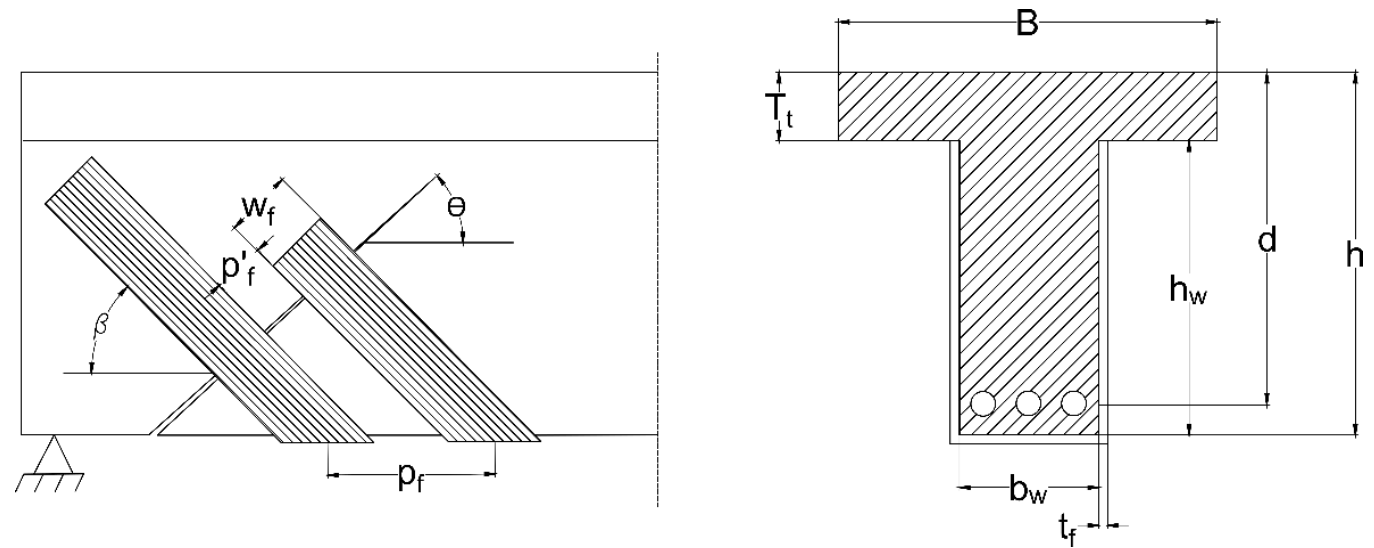

FIGURA 1: Parâmetros do reforço com FRP em faixas FONTE: CNR-DT200 (2004).

O comprimento efetivo de ancoragem é calculado adotando-se a Equação 12:

$$
l_{e}=\sqrt{\frac{E_{f} \cdot t_{f}}{2 \cdot f_{m t m}}}
$$

Em que:

$E_{f}=$ módulo da fibra de carbono (MPa);

$\boldsymbol{t}_{f}=$ espessura da fibra de carbono $(\mathrm{mm})$;

$\boldsymbol{f}_{m t m}=$ resistência a tração do concreto ( $\left.\mathrm{MPa}\right)$.

A resistência de descolamento de projeto para o reforço $f_{f d d}$ é descrito na Equação 13:

$$
f_{f d d}=\frac{1}{\gamma_{f d} \cdot \sqrt{\gamma_{m}}} \cdot \sqrt{\frac{2 \cdot E_{f} \cdot \Gamma_{F K}}{t_{f}}}
$$

Em que:

$\boldsymbol{V}_{f d}=$ fator de cálculo equivalente a 1,5;

$V_{m}=$ fator igual a 1,0;

$\boldsymbol{t}_{\boldsymbol{f}}=$ espessura da fibra de carbono $(\mathrm{mm})$;

$\boldsymbol{E}_{f}=$ módulo da fibra de carbono (MPa);

$\Gamma_{F K}=$ valor caraterístico da energia especifica de fratura, calculado mediante a Equação 14:

$$
\Gamma_{F K}=0.03 \cdot K_{b} \cdot \sqrt{f_{c k} \cdot f_{c t m}}
$$

$$
K_{b}=\sqrt{\frac{2-\frac{b_{f}}{b}}{1+\frac{b_{f}}{400}}} \geq 1
$$

$$
f_{c t m}=0.1 \cdot f_{c k}
$$

Em que:

$\boldsymbol{K}_{\boldsymbol{b}}=$ coeficiente geométrico;

$f_{c k}=$ resistência à compressão do concreto ( $\left.\mathrm{MPa}\right)$;

$f_{c t m}=$ resistência à tração do concreto $(\mathrm{MPa})$;

$\boldsymbol{b}_{f}=$ largura das fibras de carbono $(\mathrm{mm})$;

$\boldsymbol{b}=$ largura da seção $(\mathrm{mm})$.

\subsubsection{Modelo de Chen e Teng (2003)}

Através de uma parceria firmada entre a Universidade Politécnica de Hong Kong e a Universidade de Edimburgo, os pesquisadores propuseram um modelo baseado em uma distribuição não linear da deformação na fibra de carbono. É composto por duas diferentes abordagens para estimar a resistência, fazendo referência aos dois principais mecanismos de falha: (a) por descolamento da fibra de carbono e (b) por ruptura da fibra. A contribuição de resistência ao cortante da fibra é calculada pela Equação 17: 
$V_{f}=2 \cdot f_{f, e} \cdot t_{f} \cdot w_{f} \cdot \frac{h_{f, e} \cdot(\cot \theta+\cot \beta) \cdot \sin \beta}{s_{f}}$

Eq. [17]

Em que:

$\boldsymbol{V}_{\boldsymbol{f}}=$ valor de cortante aportado pela fibra de carbono $(\mathrm{N})$;

$\boldsymbol{f}_{f, \boldsymbol{e}}=$ tensão media efetiva na fibra de carbono (MPa);

$\boldsymbol{s}_{\boldsymbol{f}}, \boldsymbol{t}_{f}, \boldsymbol{w}_{\boldsymbol{f}}=$ espaçamento, espessura e largura das FRP $(\mathrm{mm})$;

$\boldsymbol{f}_{f, \boldsymbol{e}}=$ altura efetiva da fibra de carbono (MPa);

$\boldsymbol{\theta}=$ ângulo da fissura de falha (graus);

$\boldsymbol{\beta}=$ ângulo de inclinação do reforço de FRP (graus).

O modelo analítico de Chen e Teng (2003a; 2003b) considera que a fissura de ruptura finaliza a uma distância de $0,1 \cdot d$ da face de compressão, e define os parâmetros $\left(z_{b}, z_{t}\right)$ mostrados na Figura 2, os quais são definidos pelas Equações 18 a 20:

$$
\begin{gathered}
h_{f r p, e}=z_{b}-z_{t} \\
z_{t}=d_{f r p, t} \\
z_{b}=\left[d-\left(h-d_{f r p, t}\right)\right]-0,1 d
\end{gathered}
$$

Em que:

$\boldsymbol{h}_{f r p, e}=$ altura útil efetiva de FRP aderida às laterais da viga $(\mathrm{mm})$; $\boldsymbol{z}_{\boldsymbol{b}}=$ coordenada inferior efetiva de FRP $(\mathrm{mm})$;

$\boldsymbol{z}_{\boldsymbol{t}}=$ coordenada superior da fibra de carbono efetiva $(\mathrm{mm})$;

$\boldsymbol{d}_{f r p, t}=$ distância da face comprimida à borda superior da FRP nos lados da viga $(\mathrm{mm})$;

$\boldsymbol{d}_{\text {frp }}=$ distância da face comprimida à borda inferior da FRP nos lados da viga $(\mathrm{mm})$;

$\boldsymbol{d}=$ distância da face comprimida ao centroide da camada mais distante de aço da armadura de flexão tracionada (mm);

$\boldsymbol{h}=$ altura da viga $(\mathrm{mm})$;

$\boldsymbol{B}=$ largura de la mesa $(\mathrm{mm})$;

$\boldsymbol{b}_{\boldsymbol{w}}=$ largura da alma ( $\left.\mathrm{mm}\right)$;

$\boldsymbol{\theta}=$ ângulo da fissura principal de falha a cortante (graus);

$\beta=$ ângulo de inclinação das fibras de carbono; $\boldsymbol{T}_{f}=$ espessura da mesa $(\mathrm{mm})$.

As não linearidades para a abordagem de descolamento e ruptura são definidas dentro da tensão média efetiva na fibra de carbono, definida pela Equação 21:

$$
f_{f, e}=\sigma_{f, \max } D_{f r p}
$$

\section{Em que:}

$\boldsymbol{D}_{\text {frp }}=$ fator de distribuição de tensões na fibra de carbono;

$\sigma_{f, \text { máx }}=$ máxima tensão na fibra de carbono interceptada pela fissura de cisalhamento ( $\mathrm{MPa}$ ), sendo esta última diferente para descolamento e ruptura, descritas a seguir.
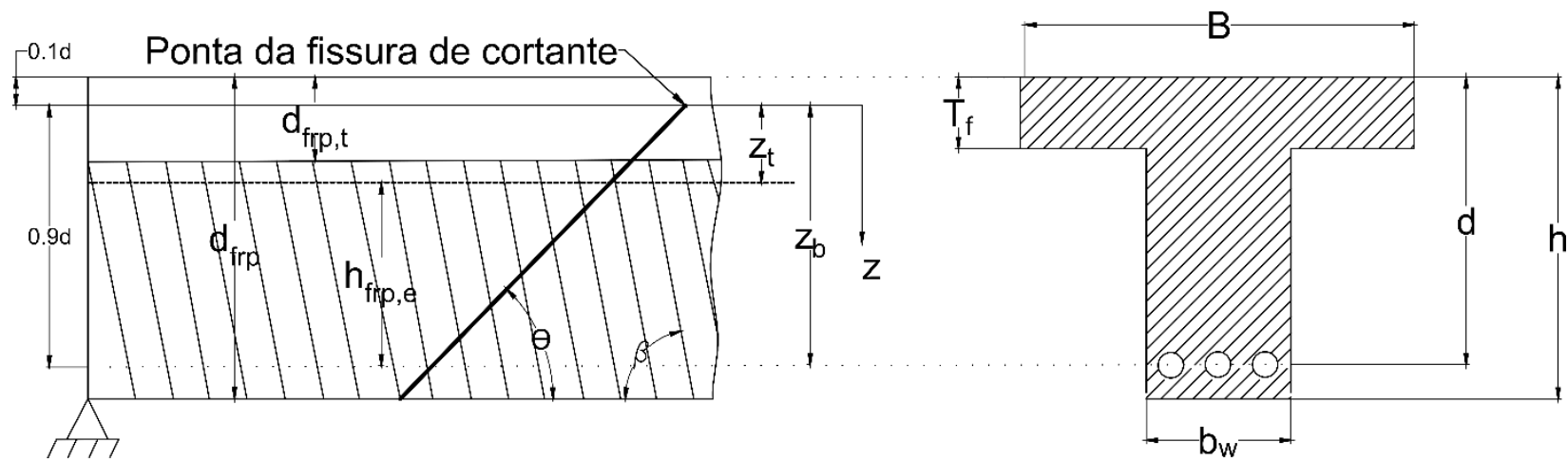

FIGURA 2: Esquema geral do modelo de Chen e Teng (2003) FONTE: Chen e Teng (2003a; 2003b). 


\subsubsection{Critério de deslocamento}

Ao aplicar-se o reforço em "U" ou lateral, levando-se em consideração o modelo de ancoragem previamente proposto por Chen e Teng (2001), prediz-se a resistência e o comprimento efetivo de aderência entre fibra de carbono e o concreto, incorporando o conceito de máxima tensão na fibra de carbono, estimado pelas Equações 22 a 27 :

$$
\begin{aligned}
& \sigma_{f, \text { máx }}=\operatorname{Min}\left[f_{f u} ; 0.427 \cdot \beta_{w} \cdot \beta_{L} \cdot \sqrt{\frac{E_{f} \cdot \sqrt{f_{c}^{\prime}}}{t_{f}}}\right] \\
& \text { Eq. [22] } \\
& \beta_{w}=\sqrt{\frac{2-w_{f} / s_{f} \cdot \operatorname{sen} \beta}{1+w_{f} / s_{f} \cdot \operatorname{sen} \beta}} \\
& \beta_{L}=\left\{\begin{array}{c}
1 \rightarrow \text { se } \quad \lambda \geq 1 \\
\operatorname{sen}(\pi \cdot \lambda / 2) \rightarrow \text { se } \quad \lambda<1
\end{array}\right.
\end{aligned}
$$

Em que:

$\sigma_{f, \text { máx }}=$ máxima tensão na fibra de carbono (MPa);

$f_{f u}=$ tensão última da fibra de carbono (MPa);

$\beta_{w}=$ efeito da fibra no concreto;

$\beta_{L}=$ efeito do comprimento de aderência;

$E_{f}=$ módulo da fibra de carbono (MPa);

$\boldsymbol{s}_{\boldsymbol{f}}, \boldsymbol{t}_{\boldsymbol{f}}, \boldsymbol{w}_{\boldsymbol{f}}=$ espaçamento, espessura e largura das FRP $(\mathrm{mm})$;

$\lambda=$ comprimento de aderência normalizado $(\mathrm{mm})$, definido pela Equação 25:

$$
\lambda=\frac{L_{\operatorname{máx}}}{L_{e}}
$$

O comprimento máximo $\left(L_{\text {máx }}\right)$ depende do tipo de reforço e o comprimento efetivo de aderência (Le) são definidos pelas Equações 26 e 27 :

$$
\begin{aligned}
& L_{\text {máx }}=h_{f e} / \operatorname{sen}(\beta) \\
& L_{e}=\sqrt{E_{f} \cdot t_{f} / \sqrt{f_{c}^{\prime}}}
\end{aligned}
$$

$\boldsymbol{L}_{e}=$ comprimento efetivo de ancoragem $(\mathrm{mm})$;

$\boldsymbol{h}_{f e}=$ altura efetiva da fibra de carbono $(\mathrm{mm})$;

$f^{\prime}{ }_{c}=$ resistência à compressão do concreto $(\mathrm{MPa})$;

$\boldsymbol{\beta}=$ ângulo de inclinação do reforço de FRP (graus).

O fator de distribuição de tensões para falha por descolamento é dado pela Equação 28:

$$
D_{f r p}\left\{\begin{array}{c}
\frac{2}{\pi \cdot \lambda} \cdot \frac{1-\cos (\pi \cdot \lambda / 2)}{\operatorname{sen}(\pi \cdot \lambda / 2)} \text { se } \lambda \leq 1 \\
1-\frac{\pi-2}{\pi \lambda} \text { se } \lambda>1
\end{array}\right.
$$

Em que:

$\boldsymbol{D}_{f r p}=$ fator de distribuição de tensões na fibra de carbono;

$\lambda=$ comprimento de aderência normalizado $(\mathrm{mm})$.

\subsubsection{Critério de ruptura}

Ao aplicar-se o reforço fechado pelo fato deste geralmente atingir o colapso da fibra de carbono como modo de ruptura a tensão máxima $\left(\sigma_{f, m a ́ x}\right)$ equivalente à tensão última na fibra de carbono e o fator de distribuição de tensões $\left(D_{f r p}\right)$ é definido pelas Equações 29 a 31:

$$
\begin{gathered}
\sigma_{f, \text { máx }}=f_{f u} \\
D_{f r p}=\frac{1+\xi}{2} \\
\xi=z_{t} / z_{b}
\end{gathered}
$$

Em que:

$\sigma_{f, \text { máx }}=$ máxima tensão na fibra de carbono (MPa); $f_{f u}=$ tensão última da fibra de carbono (MPa);

$\boldsymbol{D}_{f r p}=$ fator de distribuição de tensões na fibra de carbono;

$\xi=$ coeficiente geométrico;

$\boldsymbol{z}_{\boldsymbol{b}}=$ coordenada inferior efetiva de FRP $(\mathrm{mm})$;

$\boldsymbol{z}_{\boldsymbol{t}}=$ coordenada superior da fibra de carbono efetiva $(\mathrm{mm})$.

\subsubsection{Modelo de Chen (2010)}

$\boldsymbol{E}_{f}=$ módulo da fibra de carbono (MPa); $\boldsymbol{t}_{f}=$ espessura da fibra de carbono $(\mathrm{mm})$;
O trabalho de Chen (2010) traz um novo 
modelo de cálculo que leva em consideração o efeito de interação existente entre os estribos e as fibras de carbono através da introdução do parâmetro $K$, cujo fator incorpora o fluxo de tensões através de fatores de mobilização $K_{s}$ e $K_{f}$, para reforço com estribos e fibra de carbono, respectivamente que, por sua vez, levam em conta o efeito da interação entre os mesmos através do parâmetro $\mu$. Cabe salientar que este modelo permite avaliar a resposta do reforço em diferentes etapas da solicitação, o qual o torna um modelo bastante completo no ramo. As equações para estimativa da resistência ao cisalhamento aportada pela fibra de carbono são apresentadas mais adiante.

A resistência nominal das vigas é obtida através do princípio de superposição das contribuições de resistência do concreto, aço e a fibra de carbono, considerando-se o produto da última parcela pelo fator de interação $\mathrm{K}$, o qual leva em consideração os diversos parâmetros que influenciam no desempenho da fibra de carbono como reforço ao cisalhamento, encontrando-se descrito pela Equação 32:

$$
\begin{aligned}
& V_{u}=V_{c}+V_{s}+K V_{f} \\
& V_{f}=K \cdot V_{f, p}
\end{aligned}
$$

Em que:

$\boldsymbol{V}_{u}=$ força cortante nominal (N);

$\boldsymbol{V}_{\boldsymbol{c}}, \boldsymbol{V}_{\boldsymbol{s}}, \boldsymbol{V}_{\boldsymbol{f}}=$ contribuições de resistência do concreto, aço e a fibra de carbono, respetivamente (N).

O fator de interação (K) é definido pela Equação 34:

$$
K=K_{f}+\left(K_{s}-1\right) \cdot \mu
$$

Em que:

$\boldsymbol{K}_{f}=$ fator de mobilização da fibra de carbono;

$\boldsymbol{K}_{s}=$ fator de mobilização do aço, definido pelas Equações 35 a 37 :

$$
K_{S}=\frac{w_{e, p}^{1,4}}{\alpha+w_{e, p}^{1,4}}
$$$$
\alpha=\frac{A}{(\cos \theta)^{1,4}}
$$$$
A=\frac{4.94\left[\ln \left(h_{f, e}\right)-3.34\right] \cdot\left(f_{y}-173\right)\left(\phi_{s}+0.935\right)}{10000}
$$$$
\text { , para barras estriadas Eq. [37] }
$$ \\ Em que: \\ $\boldsymbol{h}_{f, \boldsymbol{e}}=$ altura efetiva da fibra de carbono $(\mathrm{mm})$; \\ $f_{y}=$ tensão de escoamento do aço (MPa); \\ $\phi_{s}=$ diâmetro do estribo ( $\mathrm{mm}$ ); \\ $\theta$ = ângulo da fissura principal de falha por \\ cisalhamento (graus); \\ $\alpha=$ coeficiente de cálculo; \\ $\boldsymbol{w}_{\boldsymbol{e}, \boldsymbol{p}}=$ máxima espessura da trinca $(\mathrm{mm})$.
}

O fator de mobilização da fibra de carbono

$\left(K_{f}\right)$ é definido mediante a Equação 38:

$$
K_{f}=\sigma_{f, e} / f_{f, e}
$$

Em que:

$\sigma_{f, e}=$ tensão efetiva na fibra de carbono em qualquer instante da solicitação;

$f_{f, e}=$ tensão efetiva da fibra de carbono.

Cabe salientar que o parâmetro of,e faz referência ao valor de tensão efetiva na fibra de carbono para qualquer instante, considerando-se que os fatores de mobilização não são constantes ao longo do processo de carregamento. Tal parâmetro é obtido através de simulações computacionais, apresentadas posteriormente em Chen et al. (2013), com o objetivo de gerar comparações com pesquisas experimentais. Foi considerado que a tensão ( $\sigma$ f,e) pode ser igualada à tensão efetiva na fibra de carbono para cada critério de falha (ff,e), se o fator de mobilização (Kf) for igual à unidade ( $\mathrm{Kf}$ $=1$ ). Assim, o fator de interação $(\mu)$ é calculado mediante a Equação 39:

$$
\mu=\frac{V_{s, p}}{V_{f, p}}=\frac{f_{y} A_{s v}}{f_{f, e} A_{f r p}}
$$

Em que:

$\boldsymbol{A}_{s v}=$ área dos estribos $\left(\mathrm{mm}^{2}\right)$; 
$\boldsymbol{A}_{\text {frp }}=$ área da fibra de carbono $\left(\mathrm{mm}^{2}\right)$, definida como $A_{\text {frp }}=2 \cdot \mathrm{n} \cdot \mathrm{t}_{\mathrm{f}} \cdot \mathrm{w}_{\mathrm{f}}$;

$\boldsymbol{f}_{\boldsymbol{y}}=$ tensão de escoamento do aço (MPa);

$\boldsymbol{f}_{f, e}=$ tensão efetiva na fibra de carbono, a qual depende do critério de falha do elemento.

Tendo-se o critério de descolamento e ruptura e os parâmetros abordados nas Equações 40 e 41:

$$
\begin{gathered}
f_{f, e}=\sigma_{f, \text { max }} D_{f r p} \\
\sigma_{f, \text { max }}=\min \left\{\begin{array}{c}
f_{f} \\
\sigma_{d b, \text { max }}
\end{array}\right.
\end{gathered}
$$

Em que:

$D_{\text {frp }}=$ fator de distribuição, que depende do formato de reforço utilizado;

$\boldsymbol{\sigma}_{\text {f,máx }}=$ valor da tensão máxima na fibra de carbono(MPa);

$\boldsymbol{f}_{f}=$ tensão máxima da fibra de carbono (MPa);

$\sigma_{d b, \text { máx }}=$ tensão máxima na cola (MPa).

Pelos critérios de ruptura e descolagem, tem-se que: no caso de ruptura, se igualará à capacidade máxima da fibra de carbono $\left(\sigma_{f, \text { máx }}=f_{f}\right)$, enquanto que sob o critério de descolagem, esta é calculada mediante as Equações 42 a 43:

$$
\begin{array}{cr}
\sigma_{f, \text { máx }}=\sigma_{d b, \text { máx }} & \text { Eq. [42] } \\
\sigma_{d b, \max }= \begin{cases}\sqrt{\frac{2 E_{f} G_{f}}{t_{f}}} & L_{\max } \geq L_{e} \\
\sin \left(\frac{\pi}{2} \cdot \frac{L}{L_{e}}\right) \sqrt{\frac{2 E_{f} G_{f}}{t_{f}}} & L_{\max }<L_{e}\end{cases}
\end{array}
$$$$
\text { Eq. [43] }
$$

\section{Em que:}

$\mathbf{L}_{\text {máx }}=$ comprimento máximo de ancoragem da fibra de carbono $(\mathrm{mm})$, definido pela Equação 44 :

$$
L_{\max }=\frac{h_{f, e}+h_{t}+h_{b}}{\sin \beta} \text { Para reforço em “U” Eq. [44] }
$$

Em que:

$\boldsymbol{h}_{f, \boldsymbol{e}}=$ altura efetiva da fibra de carbono $(\mathrm{mm})$; $\boldsymbol{h}_{\boldsymbol{t}}=$ altura efetiva da fibra de carbono; altura da mesa se for viga " $T$ ", se for retangular é igual a zero; $\boldsymbol{h}_{\boldsymbol{b}}=$ recobrimento inferior do aço longitudinal tracionado (mm);

$\beta=$ ângulo de inclinação das fibras de carbono (graus);

$\boldsymbol{E}_{f}=$ módulo de elasticidade da fibra de carbono (MPa);

$\boldsymbol{t}_{\boldsymbol{f}}=$ espessura da fibra de carbono $(\mathrm{mm})$;

$\boldsymbol{G}_{f}=$ energia de fratura entre superfícies;

$L_{e}=$ comprimento efetivo de ancoragem $(\mathrm{mm})$.

As Equações 45 a 54 devem ser implementadas no cálculo do fator de distribuição $\left(D_{f r p}\right)$, do qual se apresenta unicamente as expressões para reforço em faixas tipo " $U$ ".

$$
D_{f r p}=1-\left(1-\frac{\pi}{4}\right) \cdot \frac{h_{d f}}{h_{f . e}} \text { Para reforço em "U" }
$$

$$
h_{d f}=2 \delta_{f} \cdot \frac{h_{f, e}}{w_{e, p} \sin (\theta+\beta)}
$$

$$
W_{\mathrm{e}, p}=\delta_{f} \cdot \frac{1+\frac{\pi}{2} \cdot\left(\frac{h_{f, e}}{L_{e} \cdot \operatorname{sen} \beta}-1\right)}{\operatorname{sen}(\theta+\beta)}
$$

$$
\begin{gathered}
L_{e}=\sqrt{\frac{E_{f} t_{f}}{\sqrt{f_{c}^{\prime}}}} \\
\delta_{f}=2 \cdot G_{f} / \tau_{f}
\end{gathered}
$$

$$
G_{f}=0.308 \cdot \beta_{w}^{2} \cdot \sqrt{f_{t}}
$$

$$
\tau_{f}=1.5 \cdot \beta_{w} \cdot f_{t}
$$

$$
\beta_{w}=\sqrt{\frac{2-w_{f} /\left(s_{f} \sin \beta\right)}{1+w_{f} /\left(s_{f} \sin \beta\right)}}
$$




$$
\begin{gathered}
f_{t}=0.395 \cdot f_{c u}^{0.55} \\
f_{c u}=f_{c}^{\prime} / 0.8
\end{gathered}
$$

Em que:

$f_{c}=$ resistência à compressão do concreto ( $\mathrm{MPa}$ );

$f_{c u}=$ resistência à compressão do cubo de concreto (MPa);

$f_{t}=$ coeficiente de largura das faixas de fibra de carbono $\left(\mathrm{w}_{\mathrm{f}}\right)$;

$\tau_{f}, G_{f}=$ tensão máxima interfacial e energia de fratura interfacial, respetivamente e são definidos no trabalho de Lu et al. (2005);

$\boldsymbol{\beta}_{\boldsymbol{w}}=$ efeito da fibra de carbono no concreto;

$\boldsymbol{\delta}_{f}=$ deslizamento interfacial $(\mathrm{mm})$;

$L_{e}=$ comprimento efetivo de aderência $(\mathrm{mm})$;

$\boldsymbol{W}_{e, p}=$ comprimento vertical da espessura da trinca $(\mathrm{mm})$;

$\boldsymbol{\theta}=$ ângulo da fissura principal de falha por cisalhamento (graus);

$\beta$ = ângulo de inclinação das fibras de carbono (graus);

$\boldsymbol{h}_{d f}=$ distância vertical desde a ponta da trinca e o ponto de intercepção entre a frente do descolamento e a fissura de cisalhamento comprimento vertical da espessura da trinca $(\mathrm{mm})$; $\boldsymbol{h}_{f, e}=$ altura efetiva da fibra de carbono $(\mathrm{mm})$; $\boldsymbol{s}_{f}=$ espaçamento entre as fibras de carbono $(\mathrm{mm})$.

Conhecendo-se a tensão efetiva na fibra carbono, descrita pela Equação 40 sob qualquer um dos dois conceitos de falha (ruptura ou descolagem), é possível estimar a resistência aportada pela fibra de carbono mediante a Equação 55:

$$
V_{f, p}=2 \cdot f_{f, e} \cdot t_{f} \cdot w_{f} \cdot \frac{h_{f, e} \cdot(\cot \theta+\cot \beta) \cdot \sin \beta}{s_{f}}
$$

Em que:

$\boldsymbol{V}_{f, p}=$ valor de força cortante aportada pela fibra de carbono $(\mathrm{N})$;

$f_{f, e}=$ tensão media efetiva na fibra de carbono (MPa);

$\boldsymbol{s}_{\boldsymbol{f}}, \boldsymbol{t}_{\boldsymbol{f}}, \boldsymbol{w}_{\boldsymbol{f}}=$ espaçamento, espessura e largura das FRP $(\mathrm{mm})$;

$f_{f, e}=$ altura efetiva da fibra de carbono (MPa);

$\boldsymbol{\theta}=$ ângulo da fissura de falha (graus);

$\beta=$ ângulo de inclinação do reforço de FRP (graus).

\section{VALIDAÇÃO DOS MODELOS TEÓRICOS E COMPARAÇÃO COM DADOS EXPERIMENTAIS}

Os modelos apresentados para estimar as contribuições de resistência dadas pela fibra de carbono (Vf) levam em consideração diferentes parâmetros dentro das equações de cálculo, sendo destacados na Tabela 1 aqueles de mais importância em cada caso.

TABELA 1: Parâmetros considerados nos modelos teóricos.

\begin{tabular}{|c|c|c|c|c|c|c|c|}
\hline Modelo de $V_{f}$ & $\begin{array}{c}\text { Modelo de } \\
\text { colagem }\end{array}$ & $\begin{array}{c}\text { Deformação } \\
\text { da FRP }\left(\varepsilon_{f}\right)\end{array}$ & $\begin{array}{c}\text { Energia de } \\
\text { fratura }\left(G_{f}\right)\end{array}$ & $\begin{array}{c}\text { Comprimento } \\
\text { Efetivo }\left(L_{e}\right)\end{array}$ & $\begin{array}{c}\text { Relação } \\
\left(W_{f} / S_{f}\right)\end{array}$ & $\begin{array}{c}\text { Ângulo } \\
\text { de falha } \\
(\theta)\end{array}$ & $\begin{array}{c}\text { Efeito do } \\
\text { aço } \\
\text { transversal }\end{array}$ \\
\hline ACl 440.2R (2008) & $\mathrm{X}$ & $\mathrm{X}$ & - & $\mathrm{X}$ & $\mathrm{X}$ & - & - \\
\hline CNR-DT200 (2004) & $\mathrm{X}$ & $\mathrm{X}$ & - & $\mathrm{X}$ & $\mathrm{X}$ & $\mathrm{X}$ & - \\
\hline Chen et al. (2003) & $\mathrm{X}$ & - & - & $\mathrm{X}$ & $\mathrm{X}$ & $\mathrm{X}$ & - \\
\hline Chen (2010) & $\mathrm{X}$ & - & $\mathrm{X}$ & $\mathrm{X}$ & $\mathrm{X}$ & $\mathrm{X}$ & $\mathrm{X}$ \\
\hline
\end{tabular}


A validação dos diferentes modelos teóricos foi feita através da comparação das estimativas com resultados experimentais coletados a partir de uma revisão bibliográfica, considerando-se unicamente vigas reforçadas em faixas em formato de " $U$ " e com aço transversal. Os resultados experimentais e os parâmetros das vigas foram derivados dos registros das pesquisas de Chen (2010), Tumialan (2007) e Bousselham e Chaallal (2006), sendo os trabalhos originários devidamente referenciados no rodapé da Tabela 2.

$\mathrm{Na}$ Tabela 2, são apresentadas as caraterísticas geométricas e propriedades físicas dos materiais de reforço de vinte vigas de diferentes pesquisas ensaiadas experimentalmente, dentre as quais dezoito possuem seção retangular e duas têm seção transversal em " $T$ ". Todas as vigas têm em comum a característica de serem reforçadas por faixas de fibra de carbono que, como mencionado anteriormente, apresenta-se como o reforço mais eficiente em função de trabalharem isoladamente e não transferirem deformações ao sistema de reforço global e, portanto, serem mais favoráveis para no caso de falha por descolamento da fibra de carbono - que foi a ruptura registrada com maior frequência. Outra característica importante é que todas as vigas possuíam aço transversal, permitindo a aplicação dos modelos teóricos que levam em conta a interação entre os materiais de reforço.

A validação dos modelos foi realizada através do cálculo das contribuições de resistência ao cisalhamento $\left(V_{\text {fest. }}\right)$ para cada viga, comparandoos posteriormente aos valores registrados experimentalmente. A Tabela 3 apresenta os resultados obtidos para cada modelo teórico e a relação $V_{\text {fexp. }} V_{\text {fest., }}$ que permite avaliar a precisão de cada um dos modelos implementados.

Comparando-se os resultados obtidos de cada modelo teórico com os resultados experimentais pode-se observar que os obtidos através das prescrições normativas vigentes $\mathrm{ACl}$ 440.2R (2008) e CNR DT200 (2004) apresentam majoritariamente estimativas conservadoras. Embora a norma americana tenha apresentado uma relação $V_{f \text { exp }} / V_{f}$ est. média de 1,16 , nota-se que a dispersão dos resultados foi bastante considerável, gerando coeficiente de variação de $38 \%$, muito próximo da norma italiana, cujo coeficiente de variação foi de $42 \%$ para uma média de 1,93 . Tais resultados revelam médias e desvios-padrão elevados para os modelos normativos apresentados, indicando que, embora as estimativas estejam a favor da segurança, são extremamente conservadoras e antieconômicas. Por outro lado, as previsões pelos modelos propostos por Chen e Teng (2003) e Chen (2010) apresentam melhores aproximações às médias (0,94 e 1,02, respectivamente), mas coeficientes de variação ainda altos (31\% e $28 \%$, respectivamente). Esta dispersão pode dever-se ao fato dos modelos desconsiderarem os demais parâmetros que influenciam no desempenho da fibra de carbono como reforço ao cortante e necessitam ser igualmente otimizados.

A Figura 3 [a]-[d] relaciona os resultados teóricos dos modelos apresentados em relação aos experimentais, ressaltando-se os valores do coeficiente de determinação $\left(R^{2}\right)$ que representam, de maneira geral, a correlação de aproximação do modelo teórico ao experimental. Considerando-se o banco de dados experimental formado, são apresentados valores representativos do coeficiente de determinação $\left(R^{2}\right)$ para cada modelo teórico na Tabela 4.

Nota-se que o modelo proposto por Chen (2010) obteve $R^{2}=0,687$, que representa que este modelo aproxima $68,7 \%$ aos resultados registrados experimentalmente. Por se tratar de um modelo mais completo em parâmetros, também apresentou maior aproximação dentre os considerados. Já o modelo proposto por Chen et al. (2003) apresentou relativa precisão, com previsões ligeiramente mais arrojadas em termos de resistência. As estimativas segundo as normas vigentes $\mathrm{ACl} 440.2 \mathrm{R}$ (2008) e CNR DT2 (2004) foram as mais conservadoras, sobretudo esta última, que previu menores resistências e apresentou maior dispersão. A análise estatística apresentou um grau de certeza baixo, distantes em média 37,3\%, o que mostra que a correlação estabelecida ainda penaliza consideravelmente as estimativas em todos os casos. 


\begin{tabular}{|c|c|c|c|c|c|c|c|c|c|c|c|c|c|c|c|c|c|c|c|c|c|c|}
\hline \multirow{2}{*}{$\begin{array}{c}\text { Trabalho } \\
\text { fonte }\end{array}$} & \multirow{2}{*}{$\begin{array}{l}\mathbf{N}^{\circ} \\
\text { viga }\end{array}$} & \multirow{2}{*}{ ID viga } & \multirow{2}{*}{$\begin{array}{l}\text { Resultado } \\
\text { experimental } \\
\text { (KN) }\end{array}$} & \multicolumn{5}{|c|}{ Propriedades da seção } & \multicolumn{8}{|c|}{ Propriedades da FRP } & \multicolumn{5}{|c|}{ Propriedades do aço } & \multirow{2}{*}{$\begin{array}{l}\text { Ângulo } \\
\text { da } \\
\text { fissura } \\
\theta\left(1^{\circ}\right)\end{array}$} \\
\hline & & & & $\begin{array}{c}f^{\prime} c \\
(\mathrm{Mpa})\end{array}$ & $\begin{array}{c}b_{w} \\
(\mathrm{~mm})\end{array}$ & $\begin{array}{c}\mathrm{h} \\
(\mathrm{mm})\end{array}$ & $\underset{(\mathrm{mm})}{d}$ & Seção & Tipo & Formato & $\begin{array}{c}E_{\mathrm{FRP}} \\
(\mathrm{GPa})\end{array}$ & $\begin{array}{c}t_{f} \\
(\mathrm{~mm})\end{array}$ & $\begin{array}{c}F_{f} \\
(\mathrm{MPa})\end{array}$ & $\begin{array}{c}S_{f} \\
(\mathrm{~mm})\end{array}$ & $\begin{array}{c}W_{f} \\
(\mathrm{~mm})\end{array}$ & $\begin{array}{l}\text { b } \\
\left({ }^{\circ}\right)\end{array}$ & $\begin{array}{l}\text { Tipo } \\
\text { aço }\end{array}$ & $\begin{array}{c}F_{s} \\
(\mathrm{~mm})\end{array}$ & $\begin{array}{c}\mathrm{S}_{\mathrm{s}} \\
(\mathrm{mm})\end{array}$ & $\begin{array}{c}E_{\mathrm{s}} \\
\text { (GPa) }\end{array}$ & $\begin{array}{c}\mathrm{F}_{\mathrm{y}} \\
(\mathrm{MPa})\end{array}$ & \\
\hline $\begin{array}{l}\text { Bousselham e } \\
\text { Chaallal (2004) }\end{array}$ & 1 & $\mathrm{BS2}{ }^{(1)}$ & 41,20 & 35,1 & 200 & 450 & 420 & $\mathrm{R}$ & C & $u$ & 280 & 0,11 & 2800 & 400 & 100 & 90 & C & 8 & 200 & 200 & 500 & 45 \\
\hline \multirow{5}{*}{ 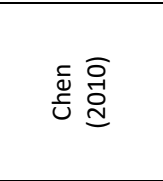 } & 2 & BS5 (2) & 33,40 & 36,0 & 200 & 450 & 390 & $R$ & C & $U$ & 233 & 0,11 & 3500 & 400 & 50 & 90 & C & 6 & 400 & 200 & 590 & 29,4 \\
\hline & 3 & PU1 ${ }^{(3)}$ & 32,50 & 40,0 & 130 & 450 & 425 & $R$ & $C$ & $U$ & 105 & 0,43 & 1400 & 200 & 40 & 90 & $\mathrm{~L}$ & 6 & 300 & 200 & 240 & 45 \\
\hline & 4 & PU2 ${ }^{(3)}$ & 20,00 & 40,0 & 130 & 450 & 425 & $\mathrm{R}$ & C & $U$ & 105 & 0,43 & 1400 & 250 & 40 & 90 & $\mathrm{~L}$ & 6 & 300 & 200 & 240 & 45 \\
\hline & 5 & PU3 ${ }^{(3)}$ & 44,50 & 40,0 & 130 & 450 & 425 & $\mathrm{R}$ & C & $U$ & 105 & 0,43 & 1400 & 300 & 40 & 45 & $\mathrm{~L}$ & 6 & 300 & 200 & 240 & 45 \\
\hline & 6 & PU4 ${ }^{(3)}$ & 40,00 & 40,0 & 130 & 450 & 425 & $R$ & $C$ & $U$ & 105 & 0,43 & 1400 & 350 & 40 & 45 & $\mathrm{~L}$ & 6 & 300 & 200 & 240 & 45 \\
\hline \multirow{8}{*}{ 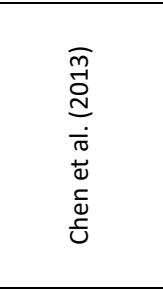 } & 7 & T6S4-90 ${ }^{(4)}$ & 85,30 & 44,1 & 140 & 600 & 520 & $T$ & C & $U$ & 230 & 0,11 & 3400 & 100 & 50 & 90 & $\mathrm{~L}$ & 6 & 400 & 200 & 520 & 27 \\
\hline & 8 & SB1-5 ${ }^{(5)}$ & 69,50 & 32,6 & 150 & 360 & 314 & $R$ & C & $U$ & 235 & 0,22 & 4200 & 120 & 40 & 90 & $\mathrm{~L}$ & 6 & 135 & 200 & 395 & 45 \\
\hline & 9 & SB1-6 ${ }^{(5)}$ & 53,50 & 32,6 & 150 & 360 & 314 & $R$ & C & $U$ & 235 & 0,22 & 4200 & 120 & 40 & 90 & $\mathrm{~L}$ & 6 & 135 & 200 & 395 & 45 \\
\hline & 10 & SB1-9 ${ }^{(5)}$ & 63,50 & 32,6 & 150 & 360 & 314 & $\mathrm{R}$ & $\mathrm{C}$ & $u$ & 235 & 0,44 & 4200 & 120 & 40 & 90 & $\mathrm{~L}$ & 6 & 135 & 200 & 395 & 45 \\
\hline & 11 & SB1-10 ${ }^{(5)}$ & 66,50 & 32,6 & 150 & 360 & 314 & $R$ & $C$ & $U$ & 235 & 0,44 & 4200 & 120 & 40 & 90 & $\mathrm{~L}$ & 6 & 135 & 200 & 395 & 45 \\
\hline & 12 & SB2-3 ${ }^{(5)}$ & 52,00 & 32,6 & 150 & 360 & 314 & $R$ & C & U & 235 & 0,22 & 4200 & 120 & 40 & 90 & $\mathrm{~L}$ & 6 & 135 & 200 & 395 & 45 \\
\hline & 13 & SB3-2 ${ }^{(5)}$ & 35,00 & 32,6 & 150 & 360 & 314 & $\mathrm{R}$ & C & $U$ & 235 & 0,22 & 4200 & 120 & 40 & 90 & $\mathrm{~L}$ & 6 & 135 & 200 & 395 & 45 \\
\hline & 14 & $2 \mathrm{~S}-\mathrm{M}(2)^{(6)}$ & 39,80 & 39,7 & 180 & 400 & 360 & $T$ & $C$ & $U$ & 218,4 & 0,352 & 2863 & 114 & 60 & 90 & $C$ & 6 & 300 & 200 & 542 & 60 \\
\hline \multirow{6}{*}{ 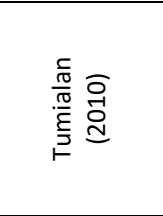 } & 15 & US45++ ${ }^{(7)}$ & 35,50 & 13,3 & 250 & 450 & 410 & $\mathrm{R}$ & $C$ & $U$ & 390 & 0,22 & 3790 & 150 & 50 & 45 & $\mathrm{C}$ & 8 & 400 & 200 & 500 & 45 \\
\hline & 16 & US45+A ${ }^{(7)}$ & 69,00 & 13,3 & 250 & 450 & 410 & $\mathrm{R}$ & C & $U$ & 390 & 0,22 & 3790 & 225 & 150 & 45 & $\mathrm{C}$ & 8 & 400 & 200 & 500 & 45 \\
\hline & 17 & US45++B ${ }^{(7)}$ & 74,00 & 13,3 & 250 & 450 & 410 & $\mathrm{R}$ & C & $U$ & 390 & 0,22 & 3790 & 225 & 150 & 45 & C & 8 & 400 & 200 & 500 & 45 \\
\hline & 18 & US $45++C^{(7)}$ & 85,00 & 13,3 & 250 & 450 & 410 & $R$ & $C$ & U & 390 & 0,22 & 3790 & 225 & 150 & 45 & $C$ & 8 & 400 & 200 & 500 & 45 \\
\hline & 19 & US45++E ${ }^{(7)}$ & 66,50 & 13,3 & 250 & 450 & 410 & $R$ & $\mathrm{C}$ & $U$ & 390 & 0,22 & 3790 & 300 & 150 & 45 & $\mathrm{C}$ & 8 & 400 & 200 & 500 & 45 \\
\hline & 20 & US45++D ${ }^{(7)}$ & 66,50 & 13,3 & 250 & 450 & 410 & $\mathrm{R}$ & $C$ & $U$ & 390 & 0,22 & 3790 & 300 & 150 & 45 & $\mathrm{C}$ & 8 & 400 & 200 & 500 & 45 \\
\hline
\end{tabular}

FONTE: Adaptado pelos autores (Convenção: Seção: R: Retangular, T: Viga "T". Tipo: C: Carbono. Formato: U: Faixa em U. Tipo aço: L: Liso; C: Corrugado)

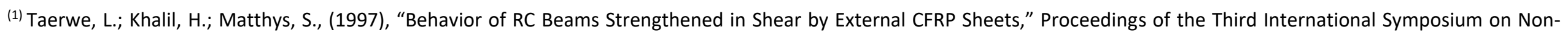
Metallic (FRP) Reinforcement for Concrete Structures, V. 1, Japan Concrete Institute, pp. 507-514.

(2) Matthys, S. (2000). Structural behaviour and design of concrete members strengthened with externally bonded FRP reinforcement. Diss. Ghent University.

(3) Diagana, C.; Li, A.; Gedalia, B.; Delmas, Y. (2003). "Shear strengthening effectiveness with CFF strips." Eng. Struct., 25(4), 507-516.

(4) Deniaud, C.; Cheng, J. J. R. (2001). "Sheer behavior of reinforced concrete T-beams with externally bonded fiber-reinforced polymer sheets." ACI Struct. J., 98(3), 386-394.

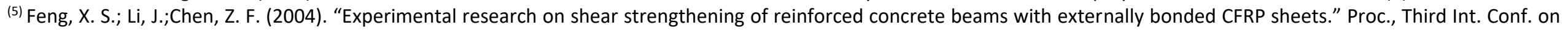
Earthquake Engineering - New Frontier and Research Transformation.

(6) Dias, S. J. E.; Barros, J. A. O. (2010). "Performance of reinforced concrete T beams strengthened in shear with NSM CFRP laminates." Eng. Struct., 32(2), 373-384.

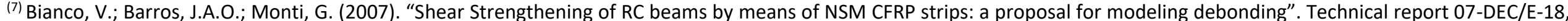
press Dep. Civil Eng., School Eng. University of Minho. 
TABELA 3: Resultados $V_{f}$ experimental por descolamento e $V_{f}$ estimado sob critério de falha por descolamento

\begin{tabular}{|c|c|c|c|c|c|c|c|c|c|c|c|}
\hline \multirow{2}{*}{$\begin{array}{c}\text { Trabalho } \\
\text { fonte }\end{array}$} & \multirow{2}{*}{$\begin{array}{l}\mathbf{N}^{\circ} \\
\text { viga }\end{array}$} & \multirow[b]{2}{*}{ ID viga } & \multirow{2}{*}{$\begin{array}{l}V_{\text {fexp. }} \\
(k N)\end{array}$} & \multicolumn{4}{|c|}{ Estimativas de resistência dos modelos ( $V_{\text {f est. }}$ ) } & \multicolumn{4}{|c|}{$V_{\text {f exp. } /} / V_{\text {f est. }}$} \\
\hline & & & & $\begin{array}{c}\mathrm{ACl} 440.2 \mathrm{R} \\
(2008)\end{array}$ & $\begin{array}{l}\text { CNR- } \\
\text { DT200 } \\
(2004)\end{array}$ & $\begin{array}{l}\text { Chen et } \\
\text { al. (2003) }\end{array}$ & $\begin{array}{l}\text { Chen } \\
(2010)\end{array}$ & $\begin{array}{c}\mathrm{ACl} 440.2 \mathrm{R} \\
(2008)\end{array}$ & $\begin{array}{c}\text { CNR-DT200 } \\
(2004)\end{array}$ & $\begin{array}{l}\text { Chen et } \\
\text { al. (2003) }\end{array}$ & $\begin{array}{l}\text { Chen } \\
\text { (2010) }\end{array}$ \\
\hline $\begin{array}{l}\text { Bousselham e } \\
\text { Chaallal (2004) }\end{array}$ & 1 & BS2 & 41,20 & 30,61 & 14,82 & 37,96 & 31,36 & 1,35 & 2,78 & 0,92 & 1,31 \\
\hline \multirow{5}{*}{ Chen (2010) } & 2 & BS5 & 33,40 & 14,13 & 12,06 & 31,39 & 26,39 & 2,36 & 2,77 & 0,94 & 1,27 \\
\hline & 3 & PU1 & 32,50 & 32,43 & 16,38 & 39,33 & 39,71 & 1,00 & 1,98 & 1,21 & 0,82 \\
\hline & 4 & PU2 & 20,00 & 25,95 & 13,10 & 32,35 & 32,67 & 0,77 & 1,53 & 1,62 & 0,61 \\
\hline & 5 & PU3 & 44,50 & 30,58 & 22,28 & 38,33 & 36,50 & 1,46 & 2,00 & 0,86 & 1,22 \\
\hline & 6 & PU4 & 40,00 & 26,21 & 19,10 & 33,48 & 31,88 & 1,53 & 2,09 & 0,84 & 1,25 \\
\hline \multirow{8}{*}{$\begin{array}{l}\text { Chen et al. } \\
\text { (2013) }\end{array}$} & 7 & T6S4-90 & 85,30 & 68,12 & 78,50 & 116,99 & 126,13 & 1,25 & 1,09 & 1,37 & 0,68 \\
\hline & 8 & SB1-5 & 69,50 & 37,74 & 18,78 & 42,90 & 41,09 & 1,84 & 3,70 & 0,62 & 1,69 \\
\hline & 9 & SB1-6 & 53,50 & 37,74 & 18,78 & 42,90 & 41,09 & 1,42 & 2,85 & 0,80 & 1,30 \\
\hline & 10 & SB1-9 & 63,50 & 53,19 & 25,27 & 57,17 & 55,81 & 1,19 & 2,51 & 0,90 & 1,14 \\
\hline & 11 & SB1-10 & 66,50 & 53,19 & 25,27 & 57,17 & 55,81 & 1,25 & 2,63 & 0,86 & 1,19 \\
\hline & 12 & SB2-3 & 52,00 & 37,74 & 18,78 & 42,90 & 41,09 & 1,38 & 2,77 & 0,83 & 1,27 \\
\hline & 13 & SB3-2 & 35,00 & 37,74 & 18,78 & 42,90 & 41,09 & 0,93 & 1,86 & 1,23 & 0,85 \\
\hline & 14 & $2 \mathrm{~S}-\mathrm{M}(2)$ & 39,80 & 64,84 & 25,96 & 37,95 & 42,71 & 0,61 & 1,53 & 0,95 & 0,93 \\
\hline \multirow{6}{*}{$\begin{array}{l}\text { Tumialan } \\
\text { (2010) }\end{array}$} & 15 & US45++ & 35,50 & 50,01 & 40,04 & 75,65 & 48,57 & 0,71 & 0,89 & 2,13 & 0,73 \\
\hline & 16 & US45+A & 69,00 & 100,02 & 71,53 & 109,50 & 91,48 & 0,69 & 0,96 & 1,59 & 0,75 \\
\hline & 17 & US45++B & 74,00 & 100,02 & 71,53 & 109,50 & 91,48 & 0,74 & 1,03 & 1,48 & 0,81 \\
\hline & 18 & US45++C & 85,00 & 100,02 & 71,53 & 109,50 & 91,48 & 0,85 & 1,19 & 1,29 & 0,93 \\
\hline & 19 & US45++E & 66,50 & 75,02 & 53,65 & 96,89 & 83,38 & 0,89 & 1,24 & 1,46 & 0,80 \\
\hline & 20 & US45++D & 66,50 & 75,02 & 53,65 & 96,89 & 83,38 & 0,89 & 1,24 & 1,46 & 0,80 \\
\hline
\end{tabular}




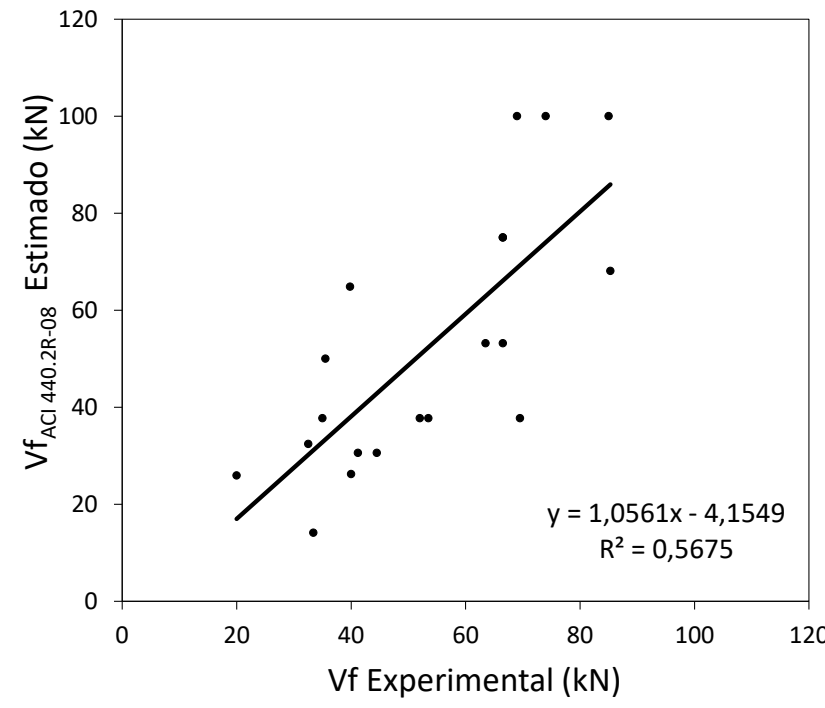

[a] Norma americana ACl 440.2R (2008)

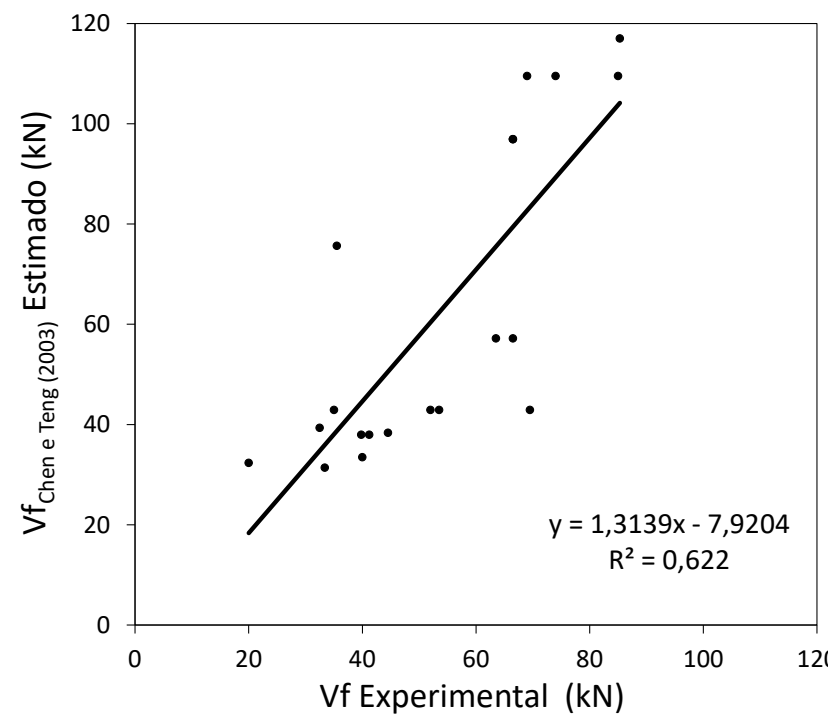

[c] Modelo de Chen et al. (2003)

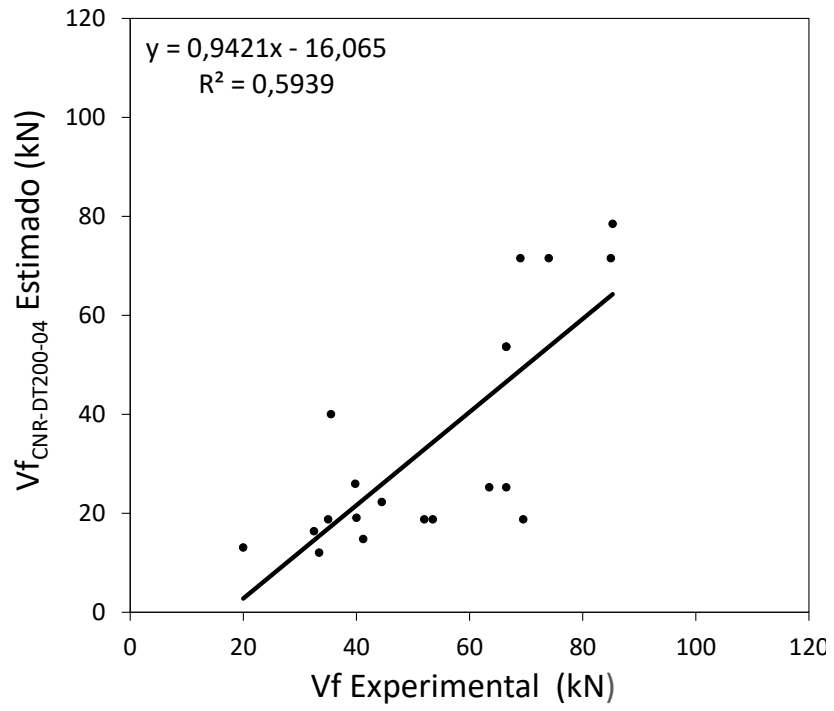

[b] Norma italiana CNR-DT200 (2004)

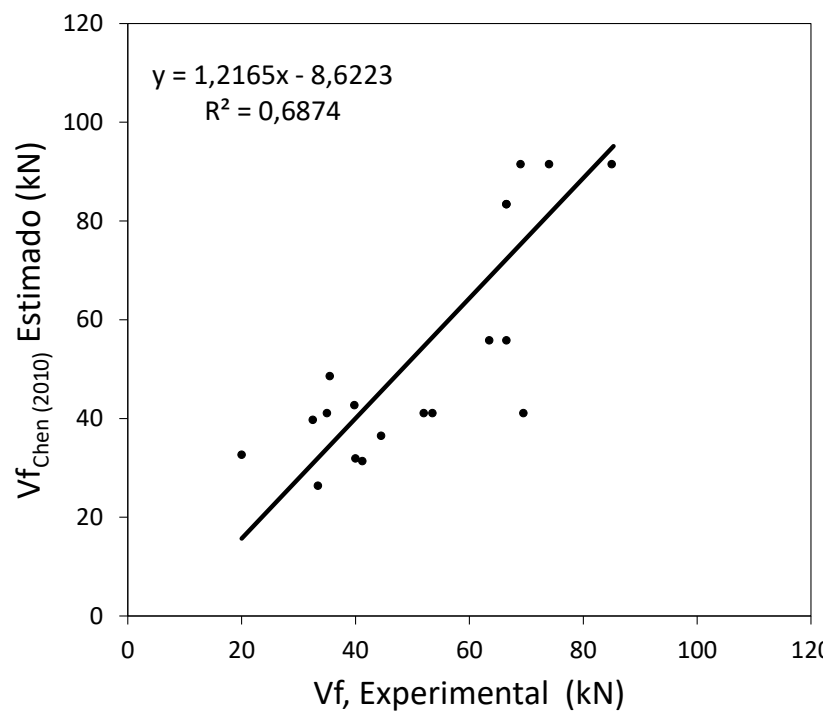

[d] Modelo de Chen (2013)

FIGURA 3: $V_{f}$ estimado $\times V_{f}$ experimental, contribuições de resistência ao cortante dada pela fibra de carbono, sendo: [a] Correlação a norma americana ACl 440.2R (2008); [b] Correlação da norma italiana CNR-DT200 (2004); [c] Correlação do modelo proposto por Chen et al. (2003); [d] Correlação do modelo proposto por Chen (2013).

FONTE: os autores.

TABELA 4: Coeficiente de determinação $\left(R^{2}\right)$ entre $V_{f}$ estimado e $V_{f}$ experimental.

\begin{tabular}{|c|c|}
\hline Modelo de $\mathbf{V}_{\mathbf{f}}$ & $\mathbf{R}^{\mathbf{2}}$ \\
\hline ACl 440.2R (2008) & 0,568 \\
\hline CNR DT2 (2004) & 0,594 \\
\hline Chen et al. (2003) & 0,622 \\
\hline Chen (2010) & 0,687 \\
\hline
\end{tabular}

FONTE: os autores. 


\section{CONCLUSÕES}

Este trabalho abordou a análise de modelos teóricos implementados conforme as prescrições normativas vigentes $\mathrm{ACl} 440.2 \mathrm{R}$ (2008) e CNR-DT200 (2004), bem como outros modelos teóricos propostos por pesquisas atuais, com a finalidade de avaliar a precisão na estimativa das contribuições de resistência ao cisalhamento devido ao sistema de reforço estrutural EB-FRP em vigas de concreto armado. A validação dos modelos teóricos foi feita a partir da comparação com resultados experimentais captados através da instrumentação dos elementos estruturais. As principais conclusões e observações do trabalho se apresentam a seguir:

(1) Os modelos estudados são baseados em premissas diferentes com respeito ao desempenho da fibra de carbono como reforço ao cisalhamento, portanto cada um destes leva em conta diferentes parâmetros na estimativa de resistência. De modo geral, conclui-se que os modelos que levam em consideração os efeitos de interação entre estribos e fibras de carbono como reforço ao cisalhamento apresentam melhor convergência com os resultados reportados experimentalmente.

(2) Existem interações entre o aço transversal e a fibra de carbono como material de reforço ao cisalhamento, cujos efeitos decorrem do desenvolvimento de tensões resistentes nas fibras de carbono. É, portanto, imprescindível avaliar modelos que considerem tal efeito. Nos modelos teóricos abordados o trabalho de Chen (2010) avaliou-se a interação entre os materiais através de fatores de redução durante diferentes etapas do carregamento e em função das caraterísticas geométricas e físicas da armadura transversal, e consequentemente gerou os melhores resultados, chegando a ampliar o grau de certeza em 11,9\% em relação aos modelos normativos. Similarmente o modelo proposto por Chen et al. (2003) apresentou certa consistência em comparação com os resultados experimentais, embora sua aproximação tenha sido em menor proporção $(5,4 \%)$.

(3) As contribuições determinadas pelos modelos teóricos das normas atuais $\mathrm{ACl}$ 440.2R (2008) e CNR DT-200 (2004) foram próximas entre si, apresentando valores próximos aos reportados pelos ensaios experimentais, embora ainda bastante dispersos. Ratifica-se, portanto, a necessidade de gerar mais estudos que permitam otimizar os códigos de projeto de estruturas reforçadas com sistemas EB-FRP.

(4) A análise de vigas reforçadas ao cisalhamento através de sistemas EB-CFRP depende de um grande número de parâmetros físicos e geométricos, tais como: altura da viga, altura efetiva do reforço, rigidez relativa da fibra de carbono, taxas de aço transversal, modelo de coesão considerado, relação geométrica de carga $(a / l)$, etc. Evidencia-se, assim, a necessidade de validação de resultados teóricos compatíveis com os dados experimentais, de maneira que as características das vigas permitam comparações confiáveis às contribuições da fibra de carbono.

(5) O desenvolvimento deste trabalho permitiu captar a importância da consideração do efeito negativo de interação entre estribos e fibras de carbono como reforço ao cisalhamento a partir da comparação entre resultados numéricos e experimentais. Dessa forma, salienta-se a necessidade do refinamento dos modelos implementados pelas normas vigentes no projeto de estruturas reforçadas com sistemas de reforço estrutural EB-FRP, o que inclui modelagem numérica em elementos finitos para considerar tais efeitos na análise.

\section{AGRADECIMENTOS}

Os autores agradecem o apoio da CAPES e do CNPq no desenvolvimento deste trabalho, através da concessão de bolsas de pesquisa. 


\section{REFERÊNCIAS BIBLIOGRÁFICAS}

$\mathrm{ACl}$ - American Concrete Institute. Guide for the Design and Construction of Externally Bonded FRP Systems for Strengthening Concrete Structures (440.2R-08), Farmington Hills, MI, 2008, 76 p.

Adhikary B. B.; Mutsuyoshi, H.; Ashraf M.Shear Strengthening of Reinforced Concrete Beams Using Fiber-Reinforced Polymer Sheets with Bonded Anchorage. ACI Structural Journal, 101(5), 2004, 660-668 p.

Bianco, V.; Barros, J.A.O.; Monti, G. Shear Strengthening of RC beams by means of NSM CFRP strips: a proposal for modeling debonding. Technical report 07-DEC/E-18, press Dep. Civil Eng., School Eng. University of Minho. 2007.

Bousselham, A.; Chaallal, O. Shear-Strengthening Reinforced Concrete Beams with Fiber-Reinforced Polymer: Assessment of Influencing Parameters and Required Research. ACl Structural Journal, V. 101, N. 2, 2004, 219-227 p.

Bousselham, A.; Chaallal, O. Behavior of Reinforced Concrete T-Beams Strenghened in shear with Carbon Fiber-Reinforced Polymer; An Experimental Study. ACl Structural Journal, V. 103, N.3, 2006, 339-347 p

Chen, J. F.; Teng, J. G. Anchorage strength models for FRP and steel plates bonded to concrete. Journal. Structural Engineering., 127 (7), 2001, 784-791 p .

Chen, J. F.; Teng, J. G. Shear capacity of FRPstrengthened RC beams: FRP debonding. Construction and Building Materials, 17 (1), 2003a. 27-41p.

Chen, J. F.; Teng, J. G. Shear capacity of FRPstrengthened RC beams: Fibre reinforced polymer rupture. Journals Structural Engineering. 129 (5), 2003b, 615-625 p.

Chen, G. M. Shear behaviour and strength of RC beams shear strengthened with externally bonded FRP reinforcement. Ph.D. thesis, Dept. of Civil and Structural Engineering, Hong Kong Polytechnic University., Hong Kong ,2010, 520 p.

Chen, G. M.; Teng, J. G.; Chen, J. F. Shear Strength Model for FRP-Strengthened RC Beams with Adverse FRP-Steel Interaction. Journal of Composites for Construction, 17(1), 2013, 50-66 p.

CNR - Italian National Research Council, CNR- DT 200/2004. Guide for the Design and Construction of Externally Bonded FRP Systems for Strengthening Existing Structures, Rome, 2008, 144 p.
Deniaud, C.; Cheng, J. J. R. Sheer behavior of reinforced concrete T-beams with externally bonded fiberreinforced polymer sheets. ACI Structural Journal. 98(3). 2001, 386-394.

Diagana, C.; Li, A.; Gedalia, B.; Delmas, Y. Shear strengthening effectiveness with CFF strips. Engineering Structures. 25(4), 2003, 507-516 p.

Dias, S. J. E.; Barros, J. A. O. Performance of reinforced concrete $T$ beams strengthened in shear with NSM CFRP laminates. Engineering Structures. 32(2) 2010, 373384p.

Dirar, S.;Less, J.; Morley, C. Precracked Reinforced Concrete T-Beams Repaired in Shear with Bonded Carbon Fiber-Reinforced Polymer Sheets. ACI Structural Journal. 109(2). 2012, 215-224 p

Federation for structural concrete (fib), Bulletin 14, Externally bonded FRP reinforcement for RC structures, Lausanne, Switzerland, 2001, TG 9.3.

Feng, X. S.; Li, J.;Chen, Z. F. Experimental research on shear strengthening of reinforced concrete beams with externally bonded CFRP sheets. Proceeding of Third International Conference on Earthquake EngineeringNew Frontier and Research Transformation. 2004.

Khalifa, A.; Gold, W. J.; Nanni, A.; Abel-Aziz, M. I. Contribution of externally bonded FRP to shear capacity members. Journal of Composites for Construction. 2(4), 1998, 195-202 p.

Kim, Y.; Quinn, K.; Ghannoum, W. M.; Jirsa, J. O. Anchorage of Carbon Fiber-Reinforced Polymer on Side Faces of Reinforced Concrete Beams to Provide Continuity. ACI Struct. Journal, 2013, 1089-1098 p.

Kim, Y.; Quinn, K.; Ghannoum, W. M.; Jirsa, J. O. Strengthening of reinforced concrete T-beams using anchored CFRP materials. ACl Struct. Journal, 111(5), 2014, 1027-1036 p.

Lopes, M. T. A. Análise de Confiabilidade de Estruturas Aplicada ao Projeto de Reforço à Força Cortante de Vigas em Concreto Armado com Compósitos de Fibras de Carbono, Tese de Doutorado, Pontifícia Universidade Católica do Rio de Janeiro. 2007, 209p.

Lu, X.Z.; Teng, J.G.; Yea, L.P.; Jiang, J.J. Bond-slip models for FRP sheets/plates bonded to concrete, Engineering Structure, 27 (6), 2005, 920-937 p.

Matthys, S. Structural behaviour and design of concrete members strengthened with externally bonded FRP reinforcement. Diss. Ghent University. 2000. 
Mofidi, A.; Chaallal, O. Shear strengthening of RC beams with EB FRP: Influencing factors and conceptual debonding model. J. Compos. Constr., 15(1), 2011, 62-74 p.

Obaidat, Y. T.; Heyden, S. Dahlblom, O. The effect of CFRP / Concrete interaction interface models when modelling retrofitted RC beams with FEM, Journal of Composites and Structures, 92, 2009, $1391-1398$ p.

Obaidat, Y. T., Structural Retrofitting of concrete beams using FRP - Debonding Issues. Doctoral Theses Lund University, Lund - Sweden, 2011.

Pellegrino, C.; Modena, C. An experimentally based analytical model for the shear capacity of FRPstrengthened reinforced concrete beams. Mechanics of Composite Materials, 44(3), 2008, 231-244 p.

Taerwe, L.; Khalil, H.; Matthys, S. Behavior of RC Beams Strengthened in Shear by External CFRP Sheets. Proceedings of the Third International Symposium on Non-Metallic (FRP) Reinforcement for Concrete Structures, V. 1, Japan Concrete Institute, 1997, 507-514 p.

Tumialan, R. P. Performance evaluation of existing analytical methods to compute the shear contribution provided by externally bonded FRP sheets in concrete structures. Masters Theses. University of Missouri-Rolla. 2007, 276p. 